\title{
Alimentação complementar de lactentes em uma cidade desenvolvida no contexto de um país em desenvolvimento ${ }^{1}$
}

\author{
Julia Laura Delbue Bernardi, ${ }^{2}$ Regina Esteves Jordão ${ }^{2}$ \\ e Antônio Azevedo Barros Filho²
}

Como citar Bernardi JLD, Jordão RE, Barros Filho AA. Alimentação complementar de lactentes em uma cidade desenvolvida no contexto de um país em desenvolvimento. Rev Panam Salud Publica. 2009;26(5):405-11.

RESUMO Objetivo. Estabelecer o momento da introdução de alimentos complementares na dieta dos lactentes no Município de Campinas, Estado de São Paulo, Brasil.

Métodos. Estudo transversal com 2857 crianças menores de 2 anos sorteadas no banco de nascidos vivos (SINASC) da secretaria de saúde do município entre 2004 e 2005. As mães responderam a um questionário contendo 87 questões fechadas que coletou informações sociais, sobre aleitamento materno e sobre a época de introdução de outros alimentos.

Resultados. A caracterização das mães revelou que 13,5\% eram adolescentes, 73,1\% completaram o ensino fundamental ou médio, 45,9\% desempenhavam funções de nível técnico, $6,7 \%$ tinham profissões de nível superior e 2,7\% eram profissionais da saúde. O nível socioeconômico médio da população foi da classe C; porém, todas as classes sociais estiveram representadas. A mediana do aleitamento materno exclusivo foi de 90 dias (IC95\%: 87,6 a 92,4). Para introdução de água (IC95\%: 118,6 a 121,4) e de chá (IC95\%: 113,2 a 126,8), a mediana foi de 120 dias; da papa salgada (IC95\%: 178,6 a 181,4) e leite em pó (IC95\%: 169,0 a 191,0), de 180 dias. Antes dos 4 meses, 39,1\% haviam ingerido leite em pó e 2,1\% refrigerantes, dados que subiram para 63,2 e 69,1\%, respectivamente, com 1 ano. As mulheres que trabalhavam em profissões de nível técnico, as que estavam desempregadas e as que não tinham companheiro ofereceram leite materno por menos tempo.

Conclusões. A mediana do aleitamento materno exclusivo está distante do preconizado devido à oferta de líquidos. A introdução alimentar revelou-se precoce, principalmente para guloseimas.

Palavras-chave Dieta; consumo de alimentos; aleitamento materno; lactentes; Brasil.

A Organização Mundial da Saúde (OMS) (1) recomenda o aleitamento materno exclusivo até os 6 meses e a sua continuidade até os 24 meses ou mais,

\footnotetext{
1 Este artigo foi elaborado a partir da tese de doutorado de Julia Laura Delbue Bernardi, intitulada "Prevalência do aleitamento materno, introdução complementar de alimentos e crescimento de menores de dois anos em Campinas, São Paulo" e apresentada em 2007 à Faculdade de Ciências Médicas, Departamento de Pediatria, da Universidade Estadual de Campinas (UNICAMP).
}

devido à capacidade protetora desse leite contra a desnutrição (2), os processos alérgicos (3), a diarreia (4) e a anemia (5). A partir do $6^{\circ}$ mês, novos alimentos

\footnotetext{
2 Universidade Estadual de Campinas (UNICAMP), Faculdade de Ciências Médicas, Departamento de Pediatria, Campinas (SP), Brasil. Enviar correspondência a Antônio Azevedo Barros Filho no seguinte endereço: Caixa Postal 6111, Cidade Universitária Zeferino Vaz, UNICAMP, CEP 13083-887, Campinas, SP, Brasil. E-mail: abarros@ fcm.unicamp.br
}

devem ser gradualmente introduzidos na dieta, de forma orientada e em conjunto com o aleitamento natural (6).

O uso de outros alimentos além do leite materno antes dos 6 meses é desnecessário e pode interferir negativamente no estabelecimento dos hábitos alimentares, contribuir para a superalimentação e trazer risco para o trato digestivo, para as vias respiratórias e para a função renal (7). Alguns tipos de líquidos e semissóli- 
dos, se oferecidos precocemente, contribuem para a diminuição da sucção da criança e da produção de leite pela mãe. Do ponto de vista da maturação fisiológica e da necessidade nutricional, a oferta inoportuna de outros alimentos além do leite materno pode levar a situações de desnutrição ou à obesidade infantil (8-10).

Este estudo objetivou verificar a idade mediana no momento da introdução dos alimentos complementares na dieta dos menores de 24 meses e obter informações sobre aleitamento e hábitos alimentares no Município de Campinas, cidade com mais de 1 milhão de habitantes e com um índice de desenvolvimento humano (IDH) de 0,8 (11), um dos melhores do Brasil.

\section{MATERIAIS E MÉTODOS}

O estudo foi do tipo transversal, com amostra randomizada. As crianças foram selecionadas por meio de sorteio aleatório feito por computador com base nos dados do Sistema de Informação de Nascidos Vivos (SINASC) (12) da secretaria municipal de saúde, que informa os nascimentos ocorridos mensalmente. $\mathrm{O}$ referido banco é composto por aproximadamente 30 variáveis da declaração de nascidos vivos e contém dados sobre o nascimento da criança, características maternas e informações de localização das famílias. $\mathrm{O}$ acesso ao banco de dados foi mediante solicitação e autorização. Inicialmente, foram avaliadas as crianças de 24 a 13 meses, seguidas pelas de 12 a 1 mês.

O tamanho da amostra, fixado em 2747 lactentes, foi calculado para permitir a estimativa da duração do aleitamento materno exclusivo (1) com um erro amostral de $0,065 \%$. O sorteio era realizado para os nascidos em determinado mês sempre no mês após o nascimento.

Os estudos epidemiológicos transversais baseados em entrevistas enfrentam questões relativas à memória do entrevistado, às recusas em participar, à impossibilidade de localizar as famílias, às mudanças de endereço, ao sigilo das informações e ao tratamento dos dados. Para reduzir a probabilidade de erro, utilizamos técnicas como ressorteio dos questionários, realização de algumas entrevistas com agendamento prévio por telefone, preferencialmente com a mãe (método status quo), cuja memória provavelmente não estaria afetada em relação ao evento recente, realização de algumas entrevistas no departamento de pes- quisa da Universidade, por solicitação do responsável, envio de cartas às unidades básicas de saúde, maternidades e conselhos de bairro, informando e esclarecendo sobre a pesquisa, e realização de um número maior de sorteios do que a amostra calculada estatisticamente. Dessa forma, prevendo perdas e recusas, foram sorteados 3000 lactentes. Das famílias sorteadas, 7,3\% não foram localizadas e 2,5\% não aceitaram participar. Nenhuma criança foi eliminada do estudo por não morar no domicílio, ser adotiva, apresentar síndrome genética ou neurológica, ser prematura ou ter tido baixo e muito baixo peso ao nascer. Assim sendo, 2857 famílias fizeram parte do estudo: entre maio e outubro de 2004 foram avaliadas 741 crianças com idade entre 24 e 13 meses; entre novembro de 2004 e maio de 2005 foram avaliadas 2116 crianças com idade entre 12 e 1 mês.

As mães foram entrevistadas em suas casas, nos diversos bairros da Cidade de Campinas, Estado de São Paulo, por duplas de nutricionistas e técnicos de enfermagem previamente treinados quanto à técnica da entrevista e ao ajuste do instrumento de avaliação, por meio de um programa piloto realizado nos 3 meses que antecederam a pesquisa. Todo o processo foi acompanhado por um supervisor de campo e pelos pesquisadores. A participação foi voluntária e realizada somente após o preenchimento do termo de consentimento livre e esclarecido pelos responsáveis. O trabalho foi aprovado pelo Comitê de Ética em Pesquisa da Universidade Estadual de Campinas (UNICAMP).

As mãe responderam a um questionário estruturado, preenchido pelos entrevistadores, contendo 87 questões fechadas, dividido em três partes, com tempo médio de preenchimento de 1 hora. No primeiro bloco foram obtidas informações de identificação da criança e realizado o inquérito socioeconômico da família. No segundo, foram investigados os dados de prenatal e as condições ao nascimento do lactente. $\mathrm{O}$ terceiro bloco se refere ao inquérito dietético, com coleta de dados sobre amamentação e alimentos complementares. As informações socioeconômicas que compreenderam o primeiro bloco do questionário possibilitaram a categorização do nível social (13) nas classes A, B, C, D, E (com gradiente decrescente de poder de compra), uma vez que foram realizadas perguntas sobre as características da moradia (tipo de construção, coleta de lixo, rede de água e esgoto e rede elétrica), o status ocupacional dos pais (empregado ou desempregado), o nível de escolaridade e a profissão materna (do lar, nível superior, superior da área da saúde - médica, psicóloga, nutricionista, fonoaudióloga, fisioterapeuta, terapeuta ocupacional, enfermeira, cirurgiã-dentista, farmacêutica -, nível técnico ou baixa escolaridade ou desempregada no momento da entrevista) e o número de bens domésticos (de 0 a 4 bens, entre geladeira, freezer, fogão, TV a cores ou preto e branco, rádio, telefone fixo e celular, computador e acesso à Internet).

As condições ao nascimento (peso e comprimento, tipo de parto, cor da pele da criança informada pela mãe), tipo de aleitamento no momento da entrevista (aleitamento materno, outros leites) e época de introdução de alimentos complementares na dieta das crianças foram aspectos investigados no segundo e terceiro blocos. Perguntava-se se a criança já havia recebido aquele alimento em questão e, em caso afirmativo, qual foi a época da introdução (dias ou meses), além do tipo de dieta que o lactente consumia no momento da entrevista. As perguntas foram direcionadas quanto ao oferecimento de líquidos (água, chá, sucos, leite fluido e leite em pó), alimentos de consistência semissólida (papas salgadas, sopas) e sólida (verduras, legumes, carnes, ovos, dieta da família) e guloseimas (açúcar, salgadinhos de saquinho, doces, balas, mel, refrigerantes). Essa sequência respeitou o Guia Alimentar para Menores de Dois Anos do Ministério da Saúde (6). Para avaliação do tempo de aleitamento materno e da época de inserção de outros alimentos para os lactentes, estipularam-se categorias do aleitamento materno, segundo as recomendações (1): aleitamento materno exclusivo (AME) quando a criança recebia somente leite de peito diretamente da mama ou extraído, sem adição de nenhum outro líquido ou sólido, com exceção de gotas ou xaropes de vitaminas, minerais e medicamentos; aleitamento materno predominante (AMP), quando havia, além do leite da mãe, inclusão de líquidos como água, chá e sucos de fruta; aleitamento materno (AM), quando a criança recebia leite materno associado a alimentos sólidos e semissólidos, incluindo leite não-humano.

Para descrever o perfil da amostra segundo as variáveis do estudo, foram 
criadas tabelas de frequência das variáveis categóricas (sexo da criança, estado civil da mãe, tipo de aleitamento) com valores de frequência absoluta $(n)$ e percentual $(\%)$ e mediana das variáveis contínuas (idade e tipo de aleitamento e introdução de alimentos). Para analisar a introdução alimentar, considerando-se o tipo de alimento que a criança consumia no dia da entrevista (status quo), foi utilizada a análise de sobrevida pelo método de estimativa de Kaplan-Meier, com intervalo de confiança de 95\% (IC95\%) e significância estatística com valor de $P \leq 0,05$. Os programas utilizados na análise dos dados foram o Epi Info para construção do banco de dados e o Statistical Package for the Social Sciences (SPSS) para as análises estatísticas.

\section{RESULTADOS}

Foram avaliadas 2857 crianças menores de 2 anos, sendo $49,3 \%$ do sexo feminino. Quanto às características sociodemográficas da população, a tabela 1 mostra que $13,5 \%$ das mães eram adolescentes, $73,1 \%$ completaram o ensino fundamental ou médio, 45,9\% desempenhavam funções de nível técnico, 6,7\% tinham profissões de nível superior e $2,7 \%$ eram profissionais da saúde. O número de bens domésticos e a renda mensal prevalente de até 6 salários mínimos caracterizaram o nível socioeconômico da população na classe C (13), embora todas as classes sociais tenham estado representadas.

A tabela 2 mostra os dados de prevalência do aleitamento materno nas diferentes categorias. Considerando-se o grupo como um todo, a mediana encontrada para AME foi de 90 dias (IC95\%: 87,6 a 92,4) e para AM, de 120 dias (IC95\%: 116,9 a 123,1). Quando a mediana do aleitamento foi observada a partir das variáveis sociodemográficas, verificou-se que as mães adolescentes, assim como as de mais idade, as com profissões de nível técnico, as desempregadas no momento da entrevista ou as que não tinham companheiro ofereceram leite materno aos seus filhos por menos tempo.

A tabela 3 mostra que no primeiro mês de vida as crianças já recebiam líquidos, como água (2 453 lactentes), chá (1 877) e outros leites sem ser o do peito (leite em pó, 1497 , antes do leite fluido, para 1358 lactentes), além de carboidratos, como o mel e o açúcar, hábitos que persistiram

TABELA 1. Variáveis sociodemográficas das crianças e das famílias participantes de estudo sobre duração do aleitamento materno exclusivo em Campinas (SP), Brasil, 2004 a 2005

\begin{tabular}{|c|c|c|}
\hline Variáveis & Amostra (No.) $)^{a}$ & $\%$ \\
\hline \multicolumn{3}{|l|}{ Cor da pele da criança } \\
\hline Branca & 2117 & 74,0 \\
\hline Parda & 610 & 21,4 \\
\hline Negra & 105 & 3,7 \\
\hline Amarela & 25 & 0,9 \\
\hline \multicolumn{3}{|l|}{ Idade materna (anos) } \\
\hline$\leq 20$ & 387 & 13,5 \\
\hline 21 a 34 & 2110 & 73,9 \\
\hline$\geq 35$ & 360 & 12,6 \\
\hline \multicolumn{3}{|l|}{ Escolaridade materna } \\
\hline$\leq 4$ anos & 222 & 7,8 \\
\hline 5 a 11 anos & 2078 & 73,1 \\
\hline$\geq 12$ anos & 543 & 19,1 \\
\hline \multicolumn{3}{|l|}{ Profissão materna } \\
\hline Do lar & 1155 & 40,4 \\
\hline Nível superior & 114 & 4,0 \\
\hline Superior da área da saúde ${ }^{b}$ & 77 & 2,7 \\
\hline Nível técnico & 1310 & 45,9 \\
\hline Desempregada & 201 & 7,0 \\
\hline \multicolumn{3}{|c|}{ Renda familiar em salários mínimos ${ }^{c}$} \\
\hline$<2$ & 639 & 22,4 \\
\hline 2 a 3 & 764 & 26,7 \\
\hline 3 a 6 & 729 & 25,5 \\
\hline 6 a 10 & 277 & 9,7 \\
\hline$>10$ & 448 & 15,7 \\
\hline \multicolumn{3}{|c|}{ Número total de bens domésticos ${ }^{d}$} \\
\hline 0 a 4 & 560 & 19,6 \\
\hline 5 a 9 & 1660 & 58,1 \\
\hline 10 a 14 & 525 & 18,4 \\
\hline 15 a 19 & 103 & 3,6 \\
\hline$\geq 20$ & 9 & 0,3 \\
\hline
\end{tabular}

a Total de 2857 crianças/famílias.

b Médica, nutricionista, farmacêutica, psicóloga, cirurgiã-dentista, fonoaudióloga, terapeuta ocupacional, fisioterapeuta, enfermeira. c Salário mínimo variou entre $R \$ 260,00$ em 2004 a R $\$ 300,00$ em 2005.

d Geladeira, freezer, fogão, TV a cores ou preto/branco, rádio, telefone fixo e celular, computador e acesso a Internet.

TABELA 2. Relação entre aleitamento materno exclusivo e aleitamento materno e variáveis sociodemográficas, Campinas (SP), Brasil, 2004 a 2005

\begin{tabular}{|c|c|c|c|c|}
\hline \multirow[b]{2}{*}{ Variável } & \multicolumn{4}{|c|}{ Mediana em dias (IC95\%) } \\
\hline & & $\begin{array}{l}\text { Aleitamento } \\
\text { exclusivo }^{a}\end{array}$ & & $\begin{array}{c}\text { Aleitamento } \\
\text { materno }^{b}\end{array}$ \\
\hline \multicolumn{5}{|l|}{ Idade materna (anos) } \\
\hline$\leq 20$ & 60 & $(49,8$ a 70,2$)$ & 120 & $(109,1$ a 130,9$)$ \\
\hline 21 a 34 & 90 & $(87,2$ a 92,8$)$ & 120 & $(116,5$ a 123,5$)$ \\
\hline$\geq 35$ & 60 & $(50,9$ a 69,1$)$ & 120 & $(110,7$ a 129,3$)$ \\
\hline \multicolumn{5}{|l|}{ Profissão materna } \\
\hline Do lar & 90 & $(85,8$ a 94,2$)$ & 120 & $(113,1$ a 126,9$)$ \\
\hline Nível superior & 120 & $(94,8$ a 145,2$)$ & 120 & $(100,8$ a 139,2$)$ \\
\hline Superior da área da saúde ${ }^{c}$ & 120 & $(98,0$ a 142,0$)$ & 150 & $(134,2$ a 165,8$)$ \\
\hline Nível técnico & 60 & $(56,6$ a 63,4$)$ & 120 & $(116,1$ a 123,9$)$ \\
\hline Sem emprego & 60 & $(46,8$ a 73,2$)$ & 120 & $(110,1$ a 129,9$)$ \\
\hline \multicolumn{5}{|l|}{ Estado civil materno } \\
\hline Sem companheiro & 60 & $(56,6$ a 63,4$)$ & 120 & $(112,5$ a 127,5$)$ \\
\hline Com companheiro & 90 & $(87,2$ a 92,8$)$ & 120 & $(116,5$ a 123,5$)$ \\
\hline
\end{tabular}

a Criança recebe somente leite de peito diretamente da mama ou extraído, sem adição de nenhum outro líquido ou sólido, com exceção de gotas ou xaropes de vitaminas, minerais e medicamentos.

${ }^{b}$ Criança recebe leite materno associado a alimentos sólidos e semissólidos, incluindo leite não-humano.

${ }^{c}$ Médica, nutricionista, farmacêutica, psicóloga, cirurgiã-dentista, fonoaudióloga, terapeuta ocupacional, fisioterapeuta, enfermeira.

aos 4 e aos 6 meses, de forma crescente. A figura 1 mostra a época da introdução dos três grandes grupos alimentares, quando pelo menos $50 \%$ das crianças já tinham consumido o alimento em questão. Observa-se que os líquidos como água e chá foram os primeiros a serem oferecidos. Quanto aos alimentos 
TABELA 3. Introdução complementar de alimentos na dieta das crianças de Campinas (SP), Brasil, 2004 a 2005

\begin{tabular}{|c|c|c|c|c|c|c|c|}
\hline \multirow[b]{2}{*}{ Alimento } & \multicolumn{7}{|c|}{ Momento da introdução de complementos antes de 6 meses de vida } \\
\hline & No. & $\begin{array}{c}<1 \text { mês } \\
(\%)^{\mathrm{a}}\end{array}$ & $\begin{array}{c}4 \text { meses } \\
(\%)^{\mathrm{a}}\end{array}$ & $\begin{array}{c}6 \text { meses } \\
(\%)^{\mathrm{a}}\end{array}$ & $\begin{array}{l}\text { Mediana } \\
\text { (dias) }\end{array}$ & $\begin{array}{l}\text { Erro } \\
\text { padrão }\end{array}$ & IC95\% \\
\hline Água & 2453 & 13,5 & 61,5 & 92,4 & 120 & 0,7 & 118,6 a 121,4 \\
\hline Chá & 1877 & 20,6 & 53,5 & 67,7 & 120 & 3,5 & 113,2 a 126,8 \\
\hline Suco de frutas & 2176 & 0,8 & 44,8 & 86,4 & 150 & 0,9 & 148,3 a 151,7 \\
\hline Leite fluido & 1358 & 3,3 & 18,8 & 36,0 & 270 & 6,1 & 258,1 a 281,9 \\
\hline Leite em pó & 1497 & 16,5 & 39,1 & 51,8 & 180 & 5,6 & 169,0 a 191,0 \\
\hline Mel & 1211 & 2,6 & 16,2 & 31,4 & 300 & 6,6 & 287,1 a 312,9 \\
\hline Açúcar & 1438 & 3,2 & 20,7 & 42,5 & 240 & 6,6 & 227,2 a 252,9 \\
\hline
\end{tabular}

a Em relação ao total de cada categoria.

FIGURA 1. Função de sobrevida para introdução de alimentação complementar: guloseimas (A), líquidos (B), e alimentos sólidos e semissólidos (C), Campinas (SP), Brasil, 2004 a 2005
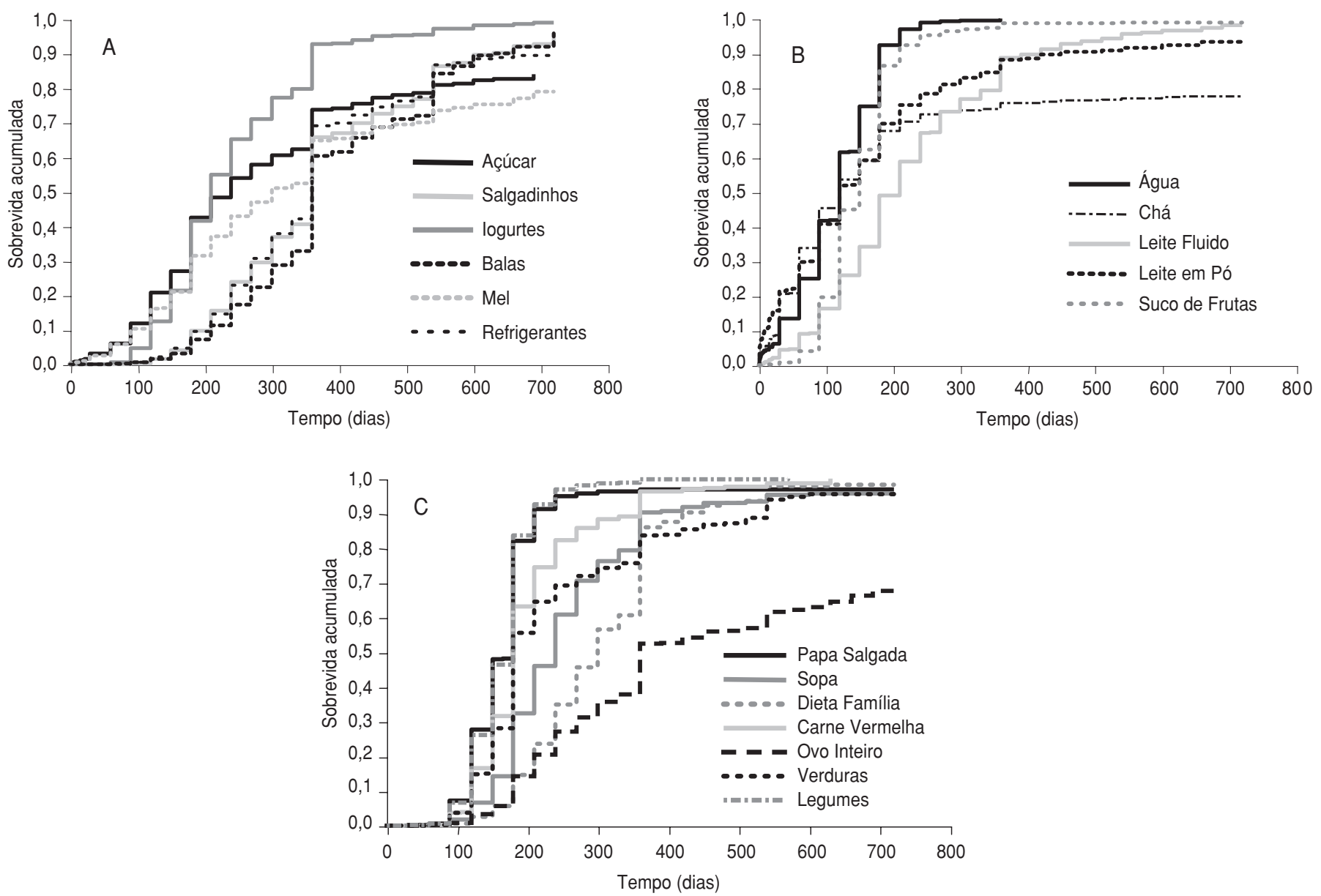

semissólidos e sólidos, a introdução da papa salgada, composta de carnes, verduras e legumes foi pioneira, com mediana de 180 dias, sendo as consistências amassada em $66,4 \%$, liquidificada em $32,9 \%$ e pedaços em $4,4 \%$. Na sequência, apareceram as sopas e a dieta da família (mediana de 240 dias e de 300 dias, res- pectivamente). A gema de ovos foi oferecida antes das claras (mediana de introdução do ovo inteiro aos 360 dias). Guloseimas como açúcar e mel foram introduzidas antes dos 10 meses de vida (medianas de 240 e 300 dias respectivamente). Salgadinhos, balas e refrigerantes foram introduzidos com 1 ano.
A tabela 4 e a figura 2 mostram a tábua de sobrevida do desmame para as categorias AME e AM em relação às variáveis sociodemográficas maternas pela análise de Kaplan-Meier, onde é possível verificar os eventos e as censuras do acontecimento. Pelo resultado, houve significância para escolaridade e estado 
TABELA 4. Análise de sobrevida do desmame para as categorias do aleitamento materno pelo método de Kaplan-Meier em relação a variáveis sociodemográficas maternas, Campinas (SP), Brasil, 2004 a 2005

\begin{tabular}{|c|c|c|c|c|c|c|c|c|c|c|}
\hline \multirow[b]{2}{*}{ Variável } & \multicolumn{5}{|c|}{ Aleitamento materno exclusivo } & \multicolumn{5}{|c|}{ Aleitamento materno } \\
\hline & No. & Mediana & $\begin{array}{c}\text { Erro } \\
\text { padrão }\end{array}$ & IC95\% & Censura $^{a}$ & No. & Mediana & $\begin{array}{c}\text { Erro } \\
\text { padrão }\end{array}$ & IC95\% & Censura $^{a}$ \\
\hline \multicolumn{11}{|l|}{ Idade (anos) } \\
\hline$<20$ & 387 & 60 & 5,2 & 49,8 a 70,2 & 30 & 382 & 120 & 5,5 & 109,1 a 130,9 & 57 \\
\hline 20 a 34 & 2110 & 90 & 1,4 & 87,2 a 92,8 & 239 & 2110 & 120 & 1,8 & 116,5 a 123,5 & 396 \\
\hline$>34$ & 360 & 60 & 4,7 & 50,9 a 69,1 & 39 & 360 & 120 & 4,7 & 110,7 a 129,3 & 59 \\
\hline & \multicolumn{5}{|c|}{ Log rank: $\chi^{2}=3,29 ; P=0,193$} & \multicolumn{5}{|c|}{ Log rank: $\chi^{2}=1,09 ; P=0,579$} \\
\hline \multicolumn{11}{|l|}{ Escolaridade (anos) } \\
\hline 0 a 4 & 222 & 90 & 6,8 & 76,7 a 103,3 & 22 & 222 & 120 & 5,1 & 110,0 a 130,1 & 44 \\
\hline 5 a 8 & 814 & 60 & 2,3 & 55,5 a 64,5 & 77 & 814 & 120 & 3,6 & 113,0 a 127,0 & 134 \\
\hline 9 a 11 & 1264 & 60 & 1,9 & 56,3 a 63,7 & 132 & 1264 & 120 & 2,3 & 115,5 a 124,5 & 228 \\
\hline$>12$ & 543 & 90 & 5,1 & 80,0 a 100,0 & 73 & 543 & 120 & 3,4 & 113,4 a 126,6 & 100 \\
\hline & \multicolumn{5}{|c|}{ Log rank: $\chi^{2}=24,91 ; P<0,001$} & \multicolumn{5}{|c|}{ Log rank: $\chi^{2}=1,78 ; P=0,618$} \\
\hline \multicolumn{11}{|c|}{ Estado civil (com companheiro) } \\
\hline Não & 482 & 60 & 1,7 & 56,6 a 63,4 & 36 & 482 & 120 & 3,8 & 112,5 a 127,5 & 63 \\
\hline $\operatorname{Sim}$ & 2375 & 90 & 1,5 & 87,2 a 92,8 & 272 & 2375 & 120 & 1,8 & 116,5 a 123,5 & 449 \\
\hline & \multicolumn{5}{|c|}{ Log rank: $\chi^{2}=20,76 ; P<0,001$} & \multicolumn{5}{|c|}{ Log rank: $\chi^{2}=13,46 ; P<0,001$} \\
\hline
\end{tabular}

a Mães que não desmamaram os seus filhos.

FIGURA 2. Função de sobrevida para desmame das categorias aleitamento materno exclusivo (AME) e aleitamento materno (AM), ${ }^{\text {a }}$ Campinas (SP), Brasil, 2004 a 2005

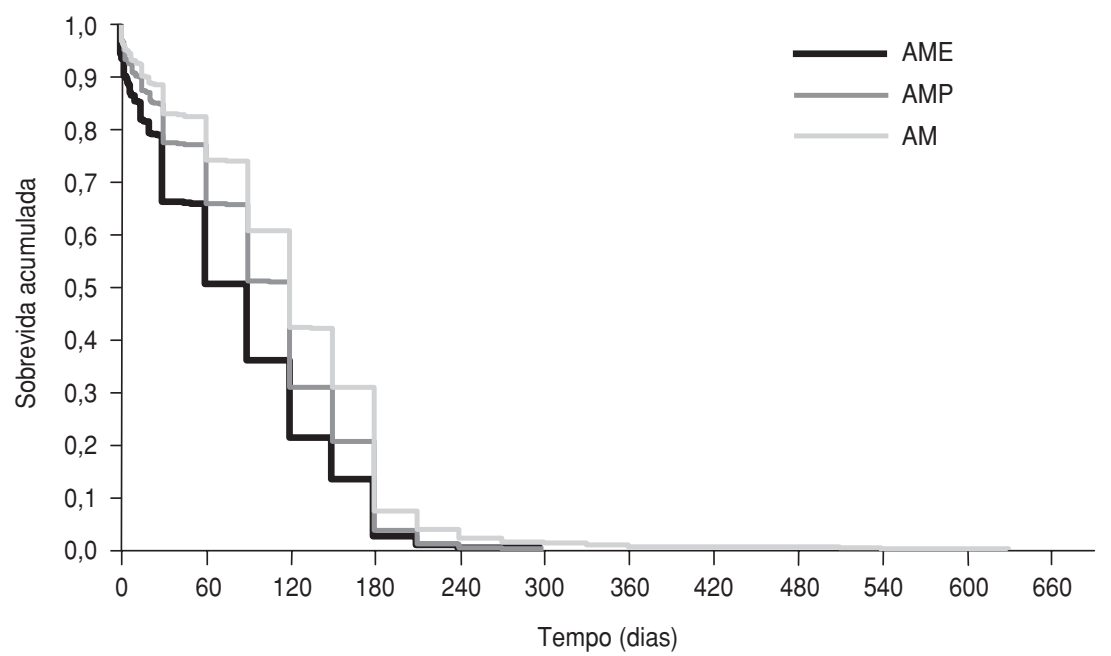

a Aleitamento exclusivo: criança recebe somente leite de peito diretamente da mama ou extraído, sem adição de nenhum outro líquido ou sólido, com exceção de gotas ou xaropes de vitaminas, minerais e medicamentos. Aleitamento materno: criança recebe leite materno associado a alimentos sólidos e semissólidos, incluindo leite não-humano. AMP = aleitamento materno predominante.

civil materno, como mostra o teste de log rank, confirmando que as mulheres que trabalhavam em profissões de nível técnico ou as que estavam desempregadas, bem como as que não tinham companheiro, ofereceram leite materno aos seus filhos por menos tempo. Neste estudo, as censuras dizem respeito às crianças que ainda estão em aleitamento no momento da entrevista. Como elas ainda não tiveram o evento (desmame), devem ser con- sideradas como censura. Observamos, na faixa etária das mães com menos de 20 anos, 30 crianças que ainda estavam em aleitamento e 357 que já haviam desmamado, ou seja, 30 censuras e 357 eventos. Na outra análise de sobrevida (figura 1), o raciocínio é outro: o evento é a introdução de determinado alimento, sendo a censura a não introdução, até aquele momento, do alimento na dieta da criança. Por isso, as curvas são ascen- dentes, enquanto são descendentes no desmame.

\section{DISCUSSÃO}

Os resultados deste estudo transversal de base populacional permitiram verificar que o aleitamento materno exclusivo está sendo interrompido na Cidade de Campinas aos 90 dias, momento no qual $50 \%$ das crianças já não recebem esse leite. Observamos ainda que a introdução complementar de alimentos não está dentro do proposto, com oferecimento precoce principalmente de guloseimas e de outros alimentos não adequados à dieta infantil. Espera-se que esses achados possam contribuir para as políticas de saúde do Município e de outras localidades com características sociodemográficas semelhantes.

Em relação ao tipo de dieta, espera-se que o viés de memória das mães tenha sido minimizado pela utilização do método status quo, que considera a mediana dos alimentos oferecidos na época da entrevista. Outro fato a ser considerado é a utilização de um banco de dados como o SINASC (12), que propiciou a localização das mães e dos lactentes. O referido banco possibilitou os cálculos estatísticos e, com $98 \%$ de cobertura, minimizou o viés pela inclusão de todas as áreas da cidade. No momento de responder o questionário, as mães disponibilizaram o cartão de nascimento e as entrevistadoras conferiram os dados. 
No estudo de Campinas, o desmame prematuro provavelmente ocorreu, entre outras razões, em virtude da introdução de água, leite em pó e chás. O chá, que se acredita ter poder calmante $(7,14)$, pode ser considerado um hábito cultural da população (66\% das mães ofereceram chá). Se introduzidos muito cedo, esses líquidos podem contribuir para a diminuição da sucção da criança e da produção de leite pela mãe, além de interromper o aleitamento exclusivo (8). Em geral, as bases do comportamento alimentar se fixam na infância, sendo transmitidas pelos pais e familiares e mantidas pela tradição, crenças e valores $(6,14,15)$.

Um estudo realizado em uma cidade da Região Sudeste (16) do Brasil mostrou que, com menos de 90 dias, 23,6\% das crianças tomavam água e $24,8 \%$ tomavam chá. Outro estudo, realizado na Região Nordeste (17), encontrou forte associação entre as condições de vida e a prática alimentar nos 2 primeiros anos de vida. Nesse estudo, as precárias condições associaram-se à interrupção precoce dos aleitamentos exclusivo e predominante. Em Campinas, observamos que até os 8 meses de idade, em média, já haviam sido introduzidos todos os grandes grupos de alimentos por nós analisados. Quanto aos anos de estudo da mãe, a interrupção precoce do aleitamento materno não mostrou associação com a baixa escolaridade. Tal fato pode ser explicado pelos bons níveis de escolaridade dessa população, já que apenas $7,8 \%$ das mães tinham menos do que 4 anos de estudo. As mães que trabalhavam em profissões de nível superior amamentaram por mais tempo.

A Pesquisa Nacional de Demografia e Saúde da Criança e da Mulher (PNDS) (18), desenvolvida no ano de 2006 pelo Ministério da Saúde do Brasil, relatou que as crianças menores de 24 meses eram amamentadas exclusivamente por 2,17 meses (mediana) e que a interrupção dessa prática ocorreu devido à introdução precoce de líquidos como água, chá e outros tipos de leites. Em Campinas, 13,5 e 20,6\% dos lactentes ingeriram água e chá, respectivamente, antes de 1 mês de vida, contribuindo, possivelmente, para o desmame exclusivo.

Com relação aos alimentos complementares, os guias alimentares (6) recomendam que a introdução seja feita após os 6 meses de idade, com uma sequência para o consumo de frutas, gema de ovos, cereais, tubérculos e hortaliças, legumi- nosas e carnes. A consistência deve progredir para alimentos sólidos e a dieta deve ser variada. Na população de Campinas, 33,1\% das mães relataram oferecer papa salgada dos 6 aos 7 meses, sendo a consistência amassada a mais prevalente, seguida da forma liquidificada. Essa prática é inadequada diante da recomendação de que seja oferecida a consistência amassada e em seguida em pedaços, mas não a líquida, que pode ser prejudicial ao processo de desenvolvimento da mastigação $(8,19)$. Antes dos 6 meses, neste estudo, observamos a introdução de chá, água e leite em pó e suco de frutas. Aos 6 meses, papa salgada, verduras, legumes e carnes; aos 7 meses, leite fluido, bolacha e pão; aos 8 meses, sopa e açúcar refinado; e aos 10 meses mel e dieta da família. Com 1 ano foi introduzido o ovo inteiro. Dessa forma, a introdução mostrou-se precoce para líquidos, mel, açúcar e guloseimas, próxima da adequação para alimentos sólidos e semissólidos e tardia para ovos. Resultados semelhantes foram encontrados em um estudo nacional (20) envolvendo duas unidades básicas de saúde, onde as carnes e os ovos foram introduzidos no final do primeiro ano de vida, bem como em um estudo internacional (21). Por outro lado, mais da metade das mães das crianças avaliadas acrescentaram papa salgada, carnes, verduras e legumes entre os 4 e os 7 meses. Portanto, pode-se considerar que esses alimentos fazem parte do hábito alimentar dos lactentes moradores da Cidade de Campinas, embora não estejam sendo oferecidos de acordo com as recomendações propostas pelo Ministério.

O Ministério da Saúde, em parceria com a Organização Pan-Americana da Saúde (OPAS), estabeleceu 10 passos para a alimentação saudável de crianças menores de 2 anos (6). O primeiro, o segundo, o quinto e o oitavo passos podem ser utilizados para comparação com o estudo de Campinas, onde o aleitamento exclusivo não foi oferecido plenamente até os 6 meses, havendo introdução precoce de líquidos. Além disso, a consistência das papas deveria ter sido espessa desde o início; e, por fim, as guloseimas, que devem ser evitadas nos primeiros anos de vida, foram introduzidas por volta de 1 ano, mostrando, portanto, que não foram seguidas as recomendações propostas.

A industrialização e a inserção da mulher no mercado de trabalho resulta- ram em alteração nos hábitos alimentares da família (22). Esse fato deve servir de alerta no que se refere ao planejamento das ações de saúde do Município, que, apesar do IDH elevado, teve associações com indicadores sociais que repercutiram na introdução alimentar, como mulheres vivendo sozinhas e cuidando da família sem o companheiro, fato que pode ter impacto na alimentação infantil.

As alterações na estrutura da dieta, associadas às mudanças econômicas $e$ sociais e suas repercussões na saúde populacional, bem como o preparo dos profissionais frente ao aconselhamento das questões ligadas à saúde (23), vêm sendo observadas em diversos países em desenvolvimento (24, 25). De acordo com a Pesquisa de Orçamentos Familiares (POF) (26), houve aumento no consumo de alimentos previamente preparados, refrigerantes e iogurtes $(99,49 \mathrm{e}$ $702 \%$ respectivamente), indicando mudanças alimentares com aumento do espaço para alimentos de preparo fácil que resultam em economia de tempo e facilitam a vida da mulher que está trabalhando. Por outro lado, os alimentos de preparo fácil implicam adversidades na alimentação da criança $(20,27,28)$. Ainda pelo último censo da POF, as frutas e os sucos naturais representaram $2,08 \%$ do total consumido de calorias, contra $13,13 \%$ para os refrigerantes do tipo guaraná, reforçando o quanto a alimentação infantil pode ser atingida.

Os presentes resultados permitiram evidenciar a associação entre a prática alimentar, o desmame precoce e as condições socioeconômicas da população de Campinas. Fica claro que a oferta precoce de doces e de refrigerantes, bem como a oferta tardia de legumes e frutas na alimentação, associadas a condições como o aumento do trabalho materno fora de casa, contribuem para a inadequação alimentar, tendo impacto, possivelmente, sobre o aumento da obesidade em nosso país. É importante que as ações de saúde do Município sejam focadas nas campanhas de incentivo ao aleitamento materno e na orientação quanto à introdução de outros alimentos na dieta infantil, principalmente para os grupos identificados como de risco.

Agradecimentos. À Fundação de Amparo à Pesquisa do Estado de São Paulo (FAPESP — processo 03/01755-3) pelo auxílio-pesquisa. 


\section{REFERÊNCIAS}

1. World Health Organization. The optimal duration of exclusive breastfeeding. Genebra: WHO; 2001. Disponível em www.who.int/ inf-pr-2001/en/note2001-07.html. Acessado em setembro de 2009. (Note for the press no. 7, 2 April of 2001).

2. Rao S, Kanade NA. Prolonged breastfeeding and malnutrition among rural Indian children below 3 years of age. Eur J Clin Nutr. 1992; 46(3):187-95.

3. Cushing AH, Samet JM, Lambert WE, Skipper BJ, Hunt WC, Young SA, et al. Breastfeeding reduces risk of respiratory illness in infants. Am J Epidemiol. 1998;147(9):863-70.

4. Asworth A, Fleachem RG. Interventions for the control of diarrhoeal diseases among young children: weaning education. Bull World Health Organ. 1985;63(6):1115-27.

5. Assis AMO, Gaudenzi EN, Gomes G, Ribeiro RC, Szarfarc SC, Souza SB. Níveis de hemoglobina, aleitamento materno e regime alimentar no primeiro ano de vida. Rev Saude Publica. 2004;38(4):543-51.

6. Brasil, Ministério da Saúde, Organização Pan-Americana da Saúde. Guia alimentar para crianças menores de dois anos. Brasília: Ministério da Saúde; 2002. (Série A Normas e manuais técnicos $\left.\mathrm{n}^{\circ} 107\right)$. Disponível em: www.opas.org.br/sistema/arquivos / Guiaaliment.pdf. Acessado em setembro de 2009.

7. Souza SL, Castro RM, Nogueira MI. Comportamento alimentar neonatal. Rev Bras Saude Matern Infant. 2003;3(3):241-6.

8. Akré J, ed. Alimentação infantil: bases fisiológicas. São Paulo: IBFAN Brasil; 1997. Disponível em http://www.ibfan.org.br/documentos/ ibfan/doc-288.pdf. Acessado em setembro de 2009.

9. Vieira GO, Silva LR, Vieira TO, Almeida JA, Cabral VA. Hábitos alimentares de crianças menores de 1 ano amamentadas e nãoamamentadas. J Pediatr (Rio J). 2004;80(5): $411-6$.
10. Devaney B, Ziegler P, Pac S, Karwe V, Barr SI Nutrient intakes of infants and toddlers. J Am Diet Assoc. 2004;104(1 Suppl 1):S14-21.

11. Prefeitura Municipal de Campinas. Origem. Disponível em: www.campinas.sp.gov.br/ campinas/campinas/origens/. Acessado em setembro de 2009.

12. Drumond EF, Machado CJ, França E. Subnotificação de nascidos vivos: procedimentos de mensuração a partir do Sistema de Informação Hospitalar. Rev Saude Publica. 2008; 42(1):55-63.

13. Instituto Brasileiro de Geografia e Estatística (IBGE). Disponível em: www.ibge.gov.br/ home/estatistica/populacao/condicaodevida/ indicadoresminimos/defaulttab.shtm. Acessado em setembro de 2005.

14. Jomori MM, Proença RPC, Calvo MCM. Determinantes da escolha alimentar: revisão. Rev Nutr. 2008;21(1):63-73.

15. Monte CM, Giugliani ER. Recomendações para alimentação complementar da criança em aleitamento materno. J Pediatr (Rio J). 2004;80(5 Suppl):S131-41.

16. Audi CAF, Correa AMS, Latorre MRDO. Alimentos complementares e fatores associados ao aleitamento materno e ao aleitamento materno exclusivo em lactentes até 12 meses de vida em Itapira, São Paulo, 1999. Rev Bras Saude Matern Infant. 2001;3(1):85-93.

17. Oliveira LPM, Assis AMO, Gomes GSS, Prado MS, Barreto ML. Duração do aleitamento materno, regime alimentar e fatores associados segundo condições de vida em Salvador, Bahia, Brasil. Cad Saude Publica. 2005;21(5):1519-30.

18. Brasil, Ministério da Saúde. Pesquisa Nacional de Demografia e Saúde da Criança e da Mulher (PNDS). Relatório final. Brasília: Ministério da Saúde, 2006. [Citado em 29 outubro 2008]. Disponível em: bvsms.saude.gov. $\mathrm{br} / \mathrm{bvs} / \mathrm{pnds} /$. Acessado em setembro de 2009.

19. Ramos M, Stein LM. Desenvolvimento do comportamento alimentar infantil. J Pediatr (Rio J). 2000;76 Suppl 3):S229-37.
20. Marchioni DM, Latorre M do R, Szarfac SC, de Souza SB. Complementary feeding: study on prevalence of food intake in two health centers of São Paulo city. Arch Latinoam Nutr. 2001;51(2):161-6.

21. WHO Multicentre Growth Reference Study Group. Complementary feeding in the WHO Multicentre Growth Reference Study. Acta Paediatr Suppl. 2006;450:27-37.

22. Parada CMGL, Carvalhaes MABL, Jamas MT. Complementary feeding practices to children during their first year of life. Rev Latino Am Enfermagem. 2007;15(2):282-9.

23. Bassichetto KC, Réa MF. Infant and young child feeding counseling: an intervention study. J Pediatr (Rio J). 2008;84(1):75-82.

24. Saldiva SRDM, Escuder MM, Mandini L, Levy RB, Venâncio SI. Práticas alimentares de crianças de 6 a 12 meses e fatores maternos associados. J Pediatr (Rio J). 2007;83(1):53-8

25. Victora CG, Matijasevich A, Santos IS, Barros AJ, Horta BL, Barros FC. Breastfeeding and feeding patterns in three birth cohorts in Southern Brazil: trends and differentials. Cad Saude Publica. 2008;24 Suppl 3:S409-16.

26. Brasil, Ministério da Saúde. Pesquisa de Orçamentos Familiares (POF). Disponível em: www. ibge.gov.br/home/estatistica/populacao/ condicaodevida/pof/2002aquisicao/default. shtm. Acessado em setembro de 2005.

27. Alves CRL, Goulart EMA, Colosimo EA, Goulart LMHF. Fatores de risco para o desmame entre usuárias de uma unidade básica de saúde de Belo Horizonte, Minas Gerais, Brasil, entre 1980 e 2004. Cad Saude Publica. 2008;24(6):1355-67.

28. Nielsen GA, Thomsen BL, Michaelsen KF. Influence of breastfeeding and complementary foods on growth between 5 and 10 months. Acta Paediatr. 1998;87(9):911-7.

Manuscrito recebido em 4 de dezembro de 2008. Aceito em versão revisada em 12 de março de 2009.
ABSTRACT

\section{Supplementary feeding of infants in a developed city within the context of a developing country}

Key words Diet; food consumption; breast feeding; infant; Brazil.
Objective. To establish the timing for introducing supplementary foods in the diet of infants from the city of Campinas, state of São Paulo, Brazil.

Methods. This cross-sectional study included 2857 children younger than 2 years of age randomly selected from the city department of health live birth database between 2004 and 2005. The mothers answered a questionnaire that included 87 closed questions designed to collect information concerning socioeconomic status, breastfeeding, and timing of introduction of supplementary foods.

Results. Assessment of the mothers revealed that $13.5 \%$ were adolescents, $73.1 \%$ had completed elementary or high school education, $45.9 \%$ had technical level jobs, $6,7 \%$ had jobs requiring a university degree, and $2.7 \%$ were health professionals. On average, participants belonged to socioeconomic class $\mathrm{C}$ (ranging from $\mathrm{A}$, highest, to $\mathrm{E}$, lowest), but all socioeconomic classes were represented. The median duration of exclusive breastfeeding was 90 days (95\%CI: 87.6-92.4). Water (95\%CI: 118.6-121.4) and tea (95CI\%: 113.2-126.8) were introduced at a median of 120 days. Semi-solid foods $(95 \%$ CI: 178.6-181,4) and powdered milk (95\%CI: 169.0-191.0) were introduced at a median of 180 days. Powdered milk and soft drinks had been ingested by $39.1 \%$ and $2.1 \%$, respectively, at 4 months, and by 63.2 and $69.1 \%$, respectively, at 1 year of age. Shorter breastfeeding duration was observed for women with technical level jobs, those who were unemployed, and those without a partner.

Conclusions. The median duration of exclusive breastfeeding was far from that recommended due to the offer of supplementary fluids. Supplementary foods were introduced precociously, especially sweets. 